\title{
CARACTERIZACIÓN GENÉTICA DEL JABALÍ DE LA ESTACIÓN BIOLÓGICA DE DOÑANA
}

\author{
GENETIC CHARACTERIZATION OF SOUTH SPAIN BOAR \\ Landi, V. ${ }^{1}$, Negro, J.J. ${ }^{2}$, Vega-Pla, J.L. ${ }^{3}$, Gortázar, C. ${ }^{2}$, García-Aznar Navajas, J.M. ${ }^{1}$, \\ Delgado Bermejo, J.V. ${ }^{1}$ y Martínez Martínez, A. ${ }^{1 *}$
}

\begin{abstract}
${ }^{1}$ Departamento de Genética. Universidad de Córdoba. Córdoba. España. *ib2mamaa@uco.es
${ }^{2}$ Estación Biológica de Doñana. Sevilla. España.

${ }^{3}$ Laboratorio de Investigación Aplicada. Ministerio de Defensa. Córdoba. España.
\end{abstract}

\section{Palabras claves adicionales}

Sus scrofa. Biodiversidad. Microsatélites.

\section{RESUMEN}

El jabalí de interés cinegético en la península Ibérica, cuenta con numerosas sub-poblaciones de Sus scrofa mediterraneus. El Parque Nacional Doñana es la reserva biológica más importante de España y la mayor superficie protegida con gran diversidad ecológica distribuida en tres tipos básicos de ecosistemas: dunas, playas y marismas en los que cohabita gran variedad de mamíferos, aves, reptiles, peces.... entre los que se cuentan poblaciones de jabalí. Para conocer su diversidad genética, en comparación con otras poblaciones españolas, se han estudiado 25 marcadores microsatélites porcinos y se ha determinado la variación genética dentro y entre poblaciones mediante el análisis de la heterocigosis $(0,53)$, número de alelos $(5,60)$, desviación del equilibrio de HW y Fis $(0,02)$.

\section{SUMMARY}

The wild boar, a cinegetic species of Iberian Peninsula, has numerous sub-populations of Sus scrofa mediterraneus. Doñana National Park is the most important biological reserves in Spain and the largest protected area with great ecological diversity, distributed on three basic types of ecosystems: sand dunes, beaches and marshes. Here live together a variety of species: mammals, birds, reptiles, fishes... and, among them, the wild boar. In order to determine the genetic diversity, also in comparison with other Spanish populations,

Presentado al Congreso SERGA (2010, Asturias).

\section{AdDitionAL KEYWORDS}

Sus scrofa. Biodiversity. Microsatellites.

we studied 25 microsatellite pigs markers and found genetic variation within and among populations by analysis of heterozygosity (0.53), number of alleles (5.60), deviation by HW equilibrium and Fis index (0.02).

\section{INTRODUCCIÓN}

El jabalí se ha mantenido por su interés cinegético en la península Ibérica y cuenta con numerosas sub-poblaciones de la subespecie Sus scrofa mediterraneus. Hasta hace poco, la reducción en el tamaño de las poblaciones y la distribución geográfica de especies de caza en Europa, eran compensadas simplemente con reintroducciones no controladas por las autoridades locales. Varias especies de mamíferos y aves fueron repobladas con animales criados en cautividad o recogidos de regiones distantes (Vernesi et al., 2003), sin una atención especial a las posibles consecuencias para los grupos autóctonos (extinción, hibridación) y las relaciones ecológicas con otras especies (Rhymer y Simberloff, 1996).

El Parque Nacional de Doñana es la reserva biológica más importante de España y la mayor área protegida (50 720 ha) cuyo reconocimiento internacional se debe principalmente a su gran diversidad ecológica, distribuida sobre tres tipos básicos de 
ecosistemas: dunas, playas y marismas en los que existe gran variedad de animales: mamíferos, aves, reptiles y peces... y entre ellos el jabalí. El objetivo de este trabajo es caracterizar genéticamente los jabalíes de la Reserva Biológica de Doñana con microsatélites para conocer su diversidad genética y compararla con jabalíes procedentes de otras partes de la península Ibérica.

\section{MATERIAL Y MÉTODOS}

Se obtuvieron 87 muestras en 11 lugares representativos del área del parque de Doñana: los Sotos, El puntal, Marismillas, Martinazo, Caño de la Raña, Punta del mal tiempo, Laguna Tío Ventero, Pozo Don Ignacio, Canaleja, Estación Biológica, Las Nuevas, Caño Limones, La Plancha, El Lucio, Agua Rubias, Cuartel del Inglesillo, las

Tabla I. Parámetros PIC, heterocigosidad esperada y observada, riqueza alélica y Fis en los marcadores moleculares utilizados. (Parameters PIC, expected and observed heterocigousisty, allelic richness and Fis index in the analyzed molecular markers).

\begin{tabular}{lcccccccc}
\hline Locus & PIC & He & Ho & NA & \multicolumn{2}{c}{ RA } \\
& & & & & $\mathrm{a}$ & $\mathrm{b}$ & $\mathrm{c}$ & Fis \\
\hline CGA & 0,76 & 0,775 & 0,768 & 22 & 9 & 16,98 & 17,14 & $0,116^{* *}$ \\
IGF1 & 0,691 & 0,718 & 0,704 & 8 & 4,424 & 6,717 & 7,248 & $-0,001$ \\
S0002 & 0,597 & 0,627 & 0,612 & 7 & 4,603 & 3,125 & 5,056 & $-0,143$ \\
S0005 & 0,045 & 0,055 & 0,05 & 5 & 1,563 & 2,739 & 2,352 & 0,185 \\
S0026 & 0,47 & 0,534 & 0,502 & 9 & 4,917 & 6,401 & 5,9 & 0,056 \\
S0068 & 0,49 & 0,516 & 0,503 & 11 & 6,768 & 5,348 & 6,987 & $0,156^{* *}$ \\
S0090 & 0,825 & 0,796 & 0,81 & 16 & 9,084 & 11,96 & 13,25 & $0,061^{*}$ \\
S0101 & 0,443 & 0,455 & 0,449 & 8 & 3,887 & 6,595 & 6,216 & 0,067 \\
S0155 & 0,696 & 0,685 & 0,691 & 11 & 5,12 & 8,538 & 7,813 & 0,033 \\
S0178 & 0,236 & 0,221 & 0,228 & 7 & 3,069 & 3,723 & 3,624 & $-0,013$ \\
S0215 & 0,381 & 0,461 & 0,421 & 7 & 1,999 & 5,43 & 4,844 & 0,042 \\
S0225 & 0,687 & 0,707 & 0,697 & 7 & 4,914 & 6,048 & 5,996 & $0,097^{*}$ \\
S0226 & 0,484 & 0,549 & 0,516 & 7 & 2,329 & 5,318 & 4,39 & 0,109 \\
S0228 & 0,103 & 0,102 & 0,103 & 6 & 1,963 & 3,113 & 2,72 & $-0,038$ \\
S0355 & 0,368 & 0,437 & 0,403 & 6 & 2,955 & 4,405 & 3,816 & 0,076 \\
S0386 & 0,538 & 0,624 & 0,581 & 11 & 5,396 & 9,59 & 9,234 & $0,224^{* *}$ \\
SW24 & 0,731 & 0,725 & 0,728 & 24 & 5,333 & 14,94 & 14,62 & $0,119^{* *}$ \\
SW72 & 0,696 & 0,734 & 0,715 & 13 & 5,157 & 9,241 & 9,275 & 0,039 \\
SW122 & 0,754 & 0,716 & 0,735 & 24 & 10,75 & 7,353 & 13,69 & 0,024 \\
SW240 & 0,737 & 0,745 & 0,741 & 14 & 7,832 & 10,89 & 10,53 & $0,098^{* *}$ \\
SW632 & 0,728 & 0,689 & 0,709 & 9 & 4 & 6,889 & 5,765 & $-0,025$ \\
SW857 & 0,459 & 0,426 & 0,442 & 7 & 4,04 & 5,618 & 5,583 & $0,211^{\star *}$ \\
SW911 & 0,718 & 0,725 & 0,721 & 10 & 3,873 & 9,183 & 8,451 & $0,074^{*}$ \\
SW936 & 0,594 & 0,6 & 0,597 & 8 & 3,962 & 6,295 & 5,53 & 0,034 \\
SW951 & 0,662 & 0,671 & 0,667 & 9 & 3,997 & 6,312 & 6,115 & 0,005 \\
Media & 0,556 & 0,572 & 0,564 & 10,64 & 4,837 & 7,31 & 7,446 & 0,028 \\
Dev.St & 0,208 & 0,202 & 0,204 & 5,461 & 2,315 & 3,519 & 3,826 & 0,071 \\
\hline PIC: Con & & & & & & & & \\
\hline
\end{tabular}

PIC: Contenido informacion polimórfica; He: eterocigosidad esperada; Ho: eterocigosidad observada; NA: número de alelos observado; RA: riqueza alélica en los jabalíes de la reserva de Doñana (a), en el resto de España (b) y en el total de las muestra(c); Fis: índice de fijación (individual fixation index on total subpopulation) después de 1000 bootstraps $(p<0.001)$. 
Gangas, Navazo de los Guardas. Además se han analizado 99 muestras de otras áreas españolas: Parque Regional de la Cuenca Alta del Manzanares (Madrid), Quinta Mora (Toledo) y Asturias. Todas las muestras proceden de animales cazados dentro de las áreas naturales y de los dos sexos en proporciones iguales. Se ha extraído el ADN de las muestras utilizando $100 \mathrm{mg}$ de tejido muscular o glandular mediante la digestión con proteinasa $\mathrm{K}$ y siguiente purificación con fenol/cloroformo (Sambrook, et al., 1989). El ADN se ha cuantificado con un espectrofotómetro NanoDrop 8000.

Se han amplificado 25 microsatélites recomendados por FAO/ISAG (2004), según el protocolo utilizado en Martínez et al. (2000). Los diferentes alelos han sido visualizados mediante electroforesis en un secuenciador automático ABI 377XL (Applied Biosystems, Foster City, CA, USA). El análisis de los fragmentos y la tipificación alélica se realizó mediante los programas informáticos Genescan Analysis ${ }^{\circledR}$ 3.1.2 y Genotyper ${ }^{\circledR}$ 2.5.2 respectivamente. Se han calculado las frecuencias alélicas, el número medio de alelos, las heterocigosis esperada y observada mediante el programa Microsatellite Toolkit (Park, 2001). Los valores de $\mathrm{F}_{\text {IS }}$ con un intervalo de confianza del $95 \%$ se han calculado con el programa informático Genetix v. 4.02 (Belkhir et al., 2003) y la prueba de equilibrio Hardy-Weinberg (HW) mediante el programa Genepop v. 3.1c (Raymond and Rousset, 1995), que aplica el test exacto de Fisher usando el método en cadena de Monte Carlo Markov (Guo and Thompson, 1992).

\section{RESULTADOSYDISCUSIÓN}

El número total de alelos por marcador fue elevado (tabla I), con un máximo de 22 (CGA) y un mínimo de 5(S005). Sin embargo mirando el valor de la riqueza alélica, que toma en cuenta el tamaño de muestra también, se observa menor variabilidad genética del jabalí de la reserva de Doñana. Todos los marcadores genéticos utilizados resultan medianamente polimórficos en la muestra total (PIC: $0,55 \pm 0,23$ ), demostrando que representan secuencias genómicas conservadas en el género Sus. En el jabalí del Parque de Doñana resulta un valor ligeramente más bajo de PIC con respecto al jabalí del resto de España $(0,49 \pm 0,2$ vs 0,61 $\pm 0,2)$. También es mas bajo el número de alelos del jabalí de Doñana que el de la población española (tabla I), resultado confirmado también con el valor de heterocigosidad observada $(0,53$ vs 0,65$)$. Seguramente estos resultados exponen no tanto una reducción de la variabilidad genética del jabalí del parque, sino su aislamiento geográfico con respecto a las otras poblaciones. Se encontró que la desviación de equilibrio de HardyWeinberg fue estadísticamente significativa ( $<<0,05)$, para los loci IGF1, SO178, SO228, SO386,SW632 mientras el jabalí del resto de España presentó casi la totalidad de los loci en desequilibrio de HW (datos no reportados). Estos resultados dependen de la gran heterogeneidad de las poblaciones muestreadas procedentes de lugares muy lejanos, en efecto, tratando las regiones de muestreo como razas, este desequilibrio desaparece (efecto Wahlund). En general se observa que con el valor de Fis, sólo 9 loci se encuentran en desequilibrio de heterocigotos, mientras la presencia de muchos valores negativos puede ser el índice de la existencia de hibridación entre poblaciones aisladas (Mathews y Porter, 1993).

Se observa (tabla II), que los jabalíes con un número de alelos más bajo, fueron los de Asturias $(4,76)$ y los Toledo los de más alelos. Los valores de Fis no diferentes de cero (excepto el jabalí de Toledo), indican la inexistencia de un exceso de homocigotos. La desviación del equilibrio de HW de todas la poblaciones, a excepción de Doñana, es debido probablemente a la gran extensión geográfica del muestreo que aporta una deficiencia virtual de heterocigotos y al muestreo casual de los animales.

En conclusión, la diversidad y extensión 
Tabla II. Parámetros de biodiversidad ${ }^{1}$ : número de alelos (NA), heterocigosidad esperada (He) y observada (Ho), índice de fijación sobre la sub-población (Fis) y valores de probabilidad por deviación del equlibrio de Hardy-Wenberg.(Biodiversity parameters: alleles number (NA), expected $(\mathrm{He})$ and observed $(\mathrm{Ho})$ heterocigousity, Fis index and HW equilibrium deviation).

\begin{tabular}{lcccccc}
\hline Población & $\mathrm{N}$ & $\mathrm{NA}$ & $\mathrm{Ho}$ & $\mathrm{He}$ & Fis (intervalos de confianza) & $\mathrm{H}$-W (p-value) \\
Doñana & 87 & 5,60 & 0,53 & 0,524 & $0,02(-0,02-0,05)$ & - \\
Madrid & 22 & 5,64 & 0,61 & 0,566 & $0,07(-0,05-0,13)$ & $*$ \\
Asturias & 22 & 4,76 & 0,55 & 0,527 & $0,04(-0,04-0,06)$ & $*$ \\
Toledo & 55 & 7,96 & 0,67 & 0,623 & $0,07(0,10-0,10)$ & $*$ \\
Total & 186 & 10,64 & 0,55 & 0,64 & $0,13(0,11-0,15)$ & $* *$ \\
\hline
\end{tabular}

N: número de muestras analizadas; ${ }^{1}$ calculados con 25 microsatélites en 4 poblaciones de jabalíes.

de la reserva biológica de Doñana ha sido un factor preponderante en la notable diferenciación de sus jabalíes, que conservan una elevada variabilidad genética pero no muy diferente de la que se observa en otras partes de España. La menor presencia de variantes alélicas, que denotan el aislamien-

\section{BIBLIOGRAFÍA}

Belkhir, K, Borsa, P., Chikhi, L., Raufaste, N. et Bonhomme, F. 2003. Genetix: 4.05 Logiciel sous WindowsTM pour la genetique des populations. Laboratoire Génome, Populations, Interactions. Adaptations, Université de Montpellier. Montpellier. France.

FAO. 2004. Secundary Guidelines for development if natural farm animals genetic resources management plans: Measurement of domestic animal diversity (MoDAD). Recommended microsatellite markers. Roma. Italy.

Guo, S.W. and Thompson, E.A. 1992. Performing the exact test of Hardy-Weinberg proportions for multiple alleles. Biometrics, 48: 361-372.

Martínez, A.M., Delgado, J.V., Rodero, A. and Vega Pla, J.L. 2000. Genetic structure of Iberian pig breed using microsatellites. Anim. Genet., 31: 295-301.

Mathews, N.E. and Porter, W.F. 1993. Effect of social structure on genetic structure of freeranging white-tailed deer in the Adirondack to geográfico de la población, es acompañada con un elevado equilibrio de HW. Sería interesante en futuros estudios medir la influencia filogenética de las otras poblaciones, incluyendo razas de cerdos domésticos que son muchas veces una especie contaminante del jabalí silvestre.

Mountains. J. Mammal., 74: 33-43.

Park, S.D.E. 2001. Trypanotolerance in West African cattle and the population genetic effects of selection. University of Dublin. Dublin.

Raymond, M. and Rousset, F. 1995. GENEPOP (Version 1.2): Population genetics software for exact test and ecumenicism. J. Hered., 86: 248249.

Rhymer, J. and Simberloff, D. 1996. Extinction by hybridization and introgression. Ann. Rev. Ecol. System., 27: 83-109.

Sambrook, J., Fritsch, E. and Maniatis, T. 1989. Molecular cloning: A laboratory manual. Cold Spring Harbor Laboratory Press. Cold Spring Harbor. NY.

Vernesi, C., Crestanello, B., Pecchioli, E., Tartari, D., Caramelli, D., Hauffe, H. and Bertorelle, G. 2003. The genetic impact of demographic decline and reintroduction in the wild boar (Sus scrofa): a microsatellite analysis. Mol. Ecol., 12: 585-595. 